\title{
EDITORIAL
}

\section{How can we reduce the mortality of invasive pneumococcal disease?}

\author{
T. Welte
}

I $\mathrm{n}$ this issue of the European Respiratory Review, LUDWIG et al. [1] present an article highlighting the continuous burden caused by invasive pneumococcal disease (IPD). Demographic changes due to the growing elderly population will further increase this problem. Bacterial infections of the upper and lower airways are most frequently caused by pneumococci in both adults and children, and pneumococci are an important cause of bacterial meningitis. Pneumococci carry a large armamentarium of pathogenesis factors enabling their translocation into the blood stream, i.e. bacteraemia, and which determine the amount of local tissue damage [2, 3]. These polygenic virulence capacities are the major cause of the high mortality of IPD. The mortality has not changed significantly in the past decades [4]. In addition to high mortality and morbidity, the IPD-induced healthcare costs are vast.

New antibiotics might not have a great effect. In some countries, the resistance of pneumococci against the recommended $\beta$-lactam antibiotics increases but, in most cases, the levels of resistance are low. However, this is irrelevant if the antibiotics are administered at a sufficiently high dose [5]. In addition, the mortality for IPD is similar in countries with high or low resistance rates.

For the same reason, an improvement in diagnostic procedures for faster detection of pneumococci might not have an important impact, since these pathogens are included in every treatment recommendation for empiric treatment of respiratory infections or meningitis.

Non-antibiotic treatment strategies, which are directed against the virulence factors of pneumococci (e.g. immunoglobulin Menriched immunoglobulins), are currently under development. It will take many years until data on the efficacy and safety are available. Therefore, vaccination seems to be the only available and applicable option to reduce the burden of pneumococcal disease. The dramatic reduction of morbidity and mortality in children aged $<5$ yrs following the introduction of the conjugated pneumococcal vaccine for infants and toddlers demonstrates the efficiency of vaccination. Furthermore, herd immunity caused a reduction in pneumococcal infections, even in non-vaccinated adults. This might be due to the enhanced

CORRESPONDENCE: T. Welte, Medizinische Hochschule Hannover, Carl-Neuberg-Str. 1, 30625 Hannover, Germany. E-mail: Welte.tobias@mh-hannover.de

PROVENANCE: Submitted article, peer reviewed. mucosal immunity in the vaccinated child that prevents the colonisation of the children's airways. The latter has previously been recognised as a major source of infection in adults [6].

Until recently, a 23-valent polysaccharide vaccine was available for adults, for which a dramatic reduction of bacteraemia rates was reported [7]. Polysaccharide vaccines do not induce mucosal immunity and, thus, do not affect carrier rates or herd immunity. In addition, the efficacy of this vaccine in the elderly with impaired immunological responses was uncertain [8]. Due to the approval of a 13-valent conjugation vaccine by the European and North American regulatory authorities (EMEA and FDA, respectively), a new and valuable vaccine is now available for adults aged $>50$ yrs. Efficiency studies to determine the reduction of the prevalence of pneumococcal infections are missing at the moment, but a large clinical trial in the Netherlands is under way and will be finished in approximately 2 yrs [9]. Studies with $>100,000$ participants are expensive and time consuming. Previous studies comparing the 7-valent conjugate with the 23-valent polysaccharide vaccine revealed a better antibody response [10]. This finding was confirmed for the 13-valent conjugate vaccine, but these results have not yet been published. Taking data on the immunogenicity of the vaccine into account provides some information on the effect of the vaccine. With these data, it seems to be justified to recommend conjugated vaccines. The first European country to give an official recommendation was Greece. A question for the future is the necessity of a parallel use of both types of vaccines. Polysaccharide vaccines affect more pneumococci serotypes, but the conjugate vaccines have more immunogenic power. A retrospective study in Australian children did not find any advantage [11].

LUDWIG et al. [1] claim that the vaccination rates in many countries are far behind the World Health Organization expectations. There might be various reasons for this observation. The knowledge about the burden of pneumococcal diseases is limited in the general population, as is the knowledge of physicians. There is no awareness about the effect of vaccination. National recommendations differ in the indications for vaccination. What is the appropriate age for vaccination? Which chronic disorders should be vaccinated in general? Success will only occur with international standardisation in combination with a public campaign demonstrating the necessity and significance of pneumococcal vaccination.

"Prevention is better than cure"; this point was made by Hippocrates more than 2,000 yrs ago and it is still true today. 


\section{STATEMENT OF INTEREST}

T. Welte has received fees for lectures from Pfizer, Sanofi Aventis, Novartis and MSD.

\section{REFERENCES}

1 Ludwig E, Bonanni $\mathrm{P}$, Rohde G, et al. The remaining challenges of pneumococcal disease in adults. Eur Respir Rev 2012; 21: 57-65.

2 van der Poll T, Opal SM. Pathogenesis, treatment, and prevention of pneumococcal pneumonia. Lancet 2009; 374: 1543-1556.

3 Mook-Kanamori BB, Geldhoff $\mathrm{M}$, van der Poll $\mathrm{T}$, et al. Pathogenesis and pathophysiology of pneumococcal meningitis. Clin Microbiol Rev 2011; 24: 557-591.

4 Welte T, Torres A, Nathwani D. Clinical and economic burden of community-acquired pneumonia among adults in Europe. Thorax 2012; 67: 71-79.

$5 \mathrm{Yu}$ VL, Chiou CC, Feldman C, et al. An international prospective study of pneumococcal bacteremia: correlation with in vitro resistance, antibiotics administered, and clinical outcome. Clin Infect Dis 2003; 37: 230-237.
6 Whitney CG, Pilishvili T, Farley MM, et al. Effectiveness of sevenvalent pneumococcal conjugate vaccine against invasive pneumococcal disease: a matched case-control study. Lancet 2006; 368 1495-1502.

7 Pletz MW, Maus U, Krug N, et al. Pneumococcal vaccines: mechanism of action, impact on epidemiology and adaption of the species. Int J Antimicrob Agents 2008; 32: 199-206.

8 Lee $\mathrm{H}, \mathrm{Nahm} \mathrm{MH,} \mathrm{Kim} \mathrm{KH}$. The effect of age on the response to the pneumococcal polysaccharide vaccine. BMC Infect Dis 2010; 10: 60.

9 Hak E, Grobbee DE, Sanders EA, et al. Rationale and design of CAPITA: a RCT of 13-valent conjugated pneumococcal vaccine efficacy among older adults. Neth J Med 2008; 66: 378-383.

10 de Roux A, Schmöle-Thoma B, Siber GR, et al. Comparison of pneumococcal conjugate polysaccharide and free polysaccharide vaccines in elderly adults: conjugate vaccine elicits improved antibacterial immune responses and immunological memory. Clin Infect Dis 2008; 46: 1015-1023.

11 O'Grady KA, Lee KJ, Carlin JB, et al. Increased risk of hospitalization for acute lower respiratory tract infection among Australian indigenous infants 5-23 months of age following pneumococcal vaccination: a cohort study. Clin Infect Dis 2010; 50: 970-978. 